V.I. Gavrilov

Nagoya Math. J.

Vol. 35 (1969), 151-157

\title{
ON A UNIQUENESS THEOREM
}

\section{I. GAVRILOV*}

1. Let $D$ be the open unit disk and $\Gamma$ be the unit circle in the complex plane, and denote by $\Omega$ the extended complex plane or the Riemann sphere.

Suppose that $\zeta \in \Gamma$. By $h(\zeta, \varphi)$ we denote the chord at $\zeta$ that makes the angle $\varphi,-\frac{\pi}{2}<\varphi<\frac{\pi}{2}$, with the radius $h(\zeta, 0)$ and by $\Delta\left(\zeta, \varphi_{1}, \varphi_{2}\right)$ the angle at $\zeta$ between the chords $h\left(\zeta, \varphi_{1}\right)$ and $h\left(\zeta, \varphi_{2}\right)$.

If $z_{1}$ and $z_{2}$ are points in $D$, the non-Euclidean distance between $z_{1}$ and $z_{2}$ will be denoted by $\sigma\left(z_{1}, z_{2}\right)$. For any $z_{0} \in D$ and any $\varepsilon>0$ we denote by $D\left(z_{0}, \varepsilon\right):\left\{z \in D ; \sigma\left(z, z_{0}\right)<\varepsilon\right\}$.

Let $f(z)$ be meromorphic in $D$ and $\rho(f(z))$ be its spherical derivative,

$$
\rho(f(z))=\frac{\left|f^{\prime}(z)\right|}{1+|f(z)|^{2}} .
$$

For any set $S$ in $D$ we put

$$
\mathfrak{M}(f, S)=\sup _{z \in S}\left[\left(1-|z|^{2}\right) \rho(f(z))\right] .
$$

A sequence of points $\left\{z_{n}\right\}, z_{n} \in D, n=1,2, \cdots$, is said to be a $P$ sequence for $f(z)$ if for each $\varepsilon>0$ and for each subsequence $\left\{z_{n_{\nu}}\right\}$, the function $f(z)$ assumes every value on $\Omega$, with at most two exceptions, infinitely often in the union of the disks $D\left(z_{n_{\nu}}, \varepsilon\right), \nu=1,2, \cdots$ (see [6]).

A necessary and sufficient condition for a sequence $\left\{z_{n}\right\}$ to be a $P$ sequence for $f(z)$ is the existance of a sequence $\left\{\varepsilon_{n}\right\}, \varepsilon_{n}>0, n=1,2, \cdots$, $\lim _{n \rightarrow \infty} \varepsilon_{n}=0$, such that

$$
\mathfrak{M}\left(f, \bigcup_{n=1}^{\infty} D\left(z_{n}, \varepsilon_{n}\right)\right)=\infty .
$$

[7].

Received October 14, 1968

* Department of Mathematics, Moscow University, USSR. Now visiting Indian Institute of Technology, Powai, Bombay-76 INDIA. 
For a function $f(z)$ meromorphic in $D$, a point $\zeta \in \Gamma$, a chord $h(\zeta, \varphi)$ at $\zeta$, an angle $\Delta\left(\zeta, \varphi_{1}, \varphi_{2}\right)$ at $\zeta$, and a sequence of points $\left\{z_{n}\right\}, z_{n} \in D, n=1$, $2, \cdots$, with a point of accummulation at $\zeta$, the cluster sets (see [8]) of $f(z)$ at $\zeta$ on $h(\zeta, \varphi), \Delta\left(\zeta, \varphi_{1}, \varphi_{2}\right),\left\{z_{n}\right\}$ will be denoted respectively, by $C(f, \zeta$, $h(\zeta, \varphi)), C\left(f, \zeta, \Delta\left(\zeta, \varphi_{1}, \varphi_{2}\right)\right)$ and $C\left(f, \zeta,\left\{z_{n}\right\}\right) ; C(f, \zeta, D)$ will stand for the global cluster set of $f(z)$ at $\zeta$.

A point $\zeta \in \Gamma$ is said to be a Fatou point of $f(z)$ if the set $\cup C(f, \zeta, \Delta$ $\left.\left(\zeta, \varphi_{1}, \varphi_{2}\right)\right)$ consists of a single value $a \in \Omega$, where the union is taken over all angles $\Delta\left(\zeta, \varphi_{1}, \varphi_{2}\right)$ at $\zeta$. The value $a$ is called the angular limit of $f(z)$ at $\zeta$. If the intersection of cluster sets $C\left(f, \zeta, \Delta\left(\zeta, \varphi_{1}, \varphi_{2}\right)\right)$ over all angles $\Delta\left(\zeta, \varphi_{1}, \varphi_{2}\right)$ at $\zeta$ coincides with $\Omega$, the point $\zeta$ is called a Plessner point of $f(z)$. The point $\zeta \in \Gamma$ is a Meier point of $f(z)$ if for each chord $h(\zeta, \varphi)$ at $\zeta$ we have $C(f, \zeta, h(\zeta, \varphi))=C(f, \zeta, D) \subset \Omega$.

2. Collingwood [3] proved the following generalisation of Lusin-Privalov's theorem.

Theorem A. Let $f(z)$ be meromorphic in $D$. If for a fixed $\varphi_{0},-\frac{\pi}{2}$ $<\varphi_{0}<\frac{\pi}{2}$, there exists a set $M$ of the second category on an arc $r \subset \Gamma$ such that $C\left(f, \zeta, h\left(\zeta, \varphi_{0}\right)\right) \neq \Omega$ at every $\zeta \in M$ and if, further, there exists $a$ value $a \in \Omega$ and a set $N$ metrically dense on $\gamma$ such that $a \in C(f, \zeta, h(\zeta, \varphi))$ on at least one $h(\zeta, \varphi)$ at every $\zeta \in N$, then $f(z) \equiv a$.

The success in this theorem is achieved because of the "regular distribution", of the family of chords $h\left(\zeta, \varphi_{0}\right)$ at the points $\zeta \in M$. This fact, that at all points $\zeta \in M$ the chords $h\left(\zeta, \varphi_{0}\right)$ makes a fixed angle with the radii, leads to the conclusion of existance of a point $\zeta_{0} \in \gamma$ at which $C\left(f, \zeta_{0}, D\right) \neq \Omega$. It in turn means that Fatou's theorem holds on a subarc $\gamma_{0}$ of $\gamma$. The second condition of theorem A implies the existance of a set $N_{0}$, mes $N_{0}>0$, such that at every $\zeta \in N_{0}$ the function $f(z)$ has the angular limit $a$.

A considerably wide extention of Collingwood's theorem has been obtained by E.P. Dolzhenko [5] who considered families of Jordan $\operatorname{arcs}\left\{L_{\varsigma}\right\}$ instead of the family of chords $\left\{h\left(\zeta, \varphi_{0}\right)\right\}$. The characteristic of "regular distribution" of a family $\left\{L_{\varsigma}\right\}$ is given in [5] in terms of the new notion of "uniform continuity", so that the family of chords $\left\{h\left(\zeta, \varphi_{0}\right)\right\}$ in theorem $A$ is a particular case of a uniform continuous family.

Moreover, Dolzhenko [5] has constructed an example which shows that 
his theorem is the best in the sense that from its conditions the term "uniform" cannot be omited. We cite Dolzhenko's example in part and in a form suitable for us.

Theorem B. There exists a function $f_{0}(z) \not \equiv 0$, holomorphic in $D$, that possesses at every point $\zeta \in \Gamma$ the following properties (i) $C\left(f, \zeta, h\left(\zeta, \varphi_{\varsigma}\right)\right)=\{0\}$ on a some chord $h\left(\zeta, \varphi_{\varsigma}\right)$, (ii) every chord $h(\zeta, \varphi),-\frac{\pi}{2}<\varphi<\frac{\pi}{2}$, contains a sequence of points $\left\{z_{n}\right\}, \lim _{n \rightarrow \infty} z_{n}=\zeta$, such that $\lim _{n \rightarrow \infty} \sigma\left(z_{n}, z_{n+1}\right)=0$ and $C\left(f, \zeta,\left\{z_{n}\right\}\right)=\{0\}$.

It follows from theorem $B$ that the conclusion of theorem $\mathrm{A}$ is not valid if the condition $C\left(f, \zeta, h\left(\zeta, \varphi_{0}\right)\right) \neq \Omega$ is replaced by the condition $C\left(f, \zeta,\left\{z_{n}\right\}\right) \neq \Omega$ even if the sequences $\left\{z_{n}\right\}$ are "thick" enough; for instance if $\lim _{n \rightarrow \infty} \sigma\left(z_{n}, z_{n+1}\right)=0$.

In a recent paper [1] Bagemihl has constructed a holomorphic function $\tilde{f}_{0}(z) \not \equiv 0$ in $D$ which possesses the property (i) in theorem $B$ and, in addition, each point $\zeta \in \Gamma$ is a Plessner point of $\tilde{f}_{0}(z)$. It is not known whether it is possible to choose functions $f_{0}(z)$ and $\tilde{f}_{0}(z)$ so that they tend to $\infty$ arbitrarily slow.

3. In this paper we prove a theorem similar to Collingwood's, which shows that uniqueness may be achieved not only by the condition of "regular distribution" of a family of chords but also by a definite restriction on the growth of a function.

We need the following-

Lemma. Let $f(z)$ be meromorphic in $D$ and suppose $\zeta \in \Gamma$. If there exists a chord $h(\zeta, \varphi)$ which contains a sequence of points $\left\{z_{n}\right\}, \lim _{n \rightarrow \infty} z_{n}=\zeta$, with the properties (i) $\lim _{n \rightarrow \infty} \sigma\left(z_{n}, z_{n+1}\right)=0$, (ii) $C\left(f, \zeta,\left\{z_{n}\right\}\right) \neq \Omega$ and in an angle $\Delta\left(\zeta, \varphi_{1}, \varphi_{2}\right)$ such that $\varphi_{1}<\varphi<\varphi_{2}$ we have $\mathfrak{M}\left(f, \Delta\left(\zeta, \varphi_{1}, \varphi_{2}\right)\right)<+\infty$, then the point $\zeta$ is not a Plessner point of $f(z)$.

Proof. Suppose that contrary to hypothesis the point $\zeta$ is a Plessner point of $f(z)$. It follows that for any $a \in \Omega$ there exists a sequence of points $\left\{z_{\nu}^{(a)}\right\}, \lim _{\nu \rightarrow \infty} z_{\nu}^{(a)}=\zeta$ on which $\lim _{\nu \rightarrow \infty} f\left(z_{\nu}^{(a)}\right)=a$, and a corresponding sequence of points $\left\{\tilde{z}_{\nu}\right\}$ on $h(\zeta, \varphi)$ such that $\lim _{\nu \rightarrow \infty} \sigma\left(z_{\nu}^{(a)}, \tilde{z}_{\nu}\right)=0$. In particular it holds for a value $a \notin C\left(f, \zeta,\left\{z_{n}\right\}\right)$. By the condition (i) of lemma, the sequence $\left\{z_{n}\right\}$ contains a subsequence $\left\{z_{n_{\nu}}\right\}$ for which $\lim _{\nu \rightarrow \infty} \sigma\left(z_{n_{\nu}}, \tilde{z}_{\nu}\right)=0$. Hence we have two sequences $\left\{z_{n_{\nu}}\right\}$ and $\left\{z_{\nu}^{(a)}\right\}$ such that $\lim _{\nu \rightarrow \infty} \sigma\left(z_{n_{\nu}}, z_{\nu}^{(a)}\right)=0$ and

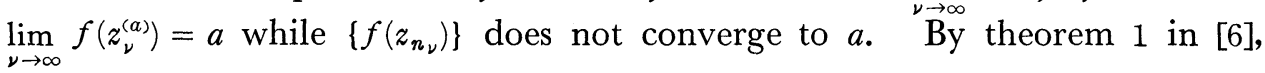


the sequence $\left\{z_{n}\right\}$ is a $P$-sequence for $f(z)$. This implies (1) which contradicts $\mathfrak{M}\left(f, \Delta\left(\zeta, \varphi_{1}, \varphi_{2}\right)\right)<+\infty$.

THeOREM 1. Let $f(z)$ be meromorphic in $D$. If at every point $\zeta$ of a set $M$ of the second category on an arc $\gamma \subset \Gamma$ there exists a chord $h\left(\zeta, \varphi_{\varsigma}\right)$ such that it contains a sequence of points $\left\{z_{n}\right\}, \lim _{n \rightarrow \infty} z_{n}=\zeta$, for which (i) $\lim _{n \rightarrow \infty} \sigma\left(z_{n}, z_{n+1}\right)=0$ (ii) $C\left(f, \zeta,\left\{z_{n}\right\}\right) \neq \Omega$ and (iii) in an angle $\Delta\left(\zeta, \varphi_{\varsigma}^{1}, \varphi_{\varsigma}^{2}\right),-\frac{\pi}{2}<\varphi_{\varsigma}^{1}<\varphi_{\varsigma}<\varphi_{\varsigma}^{2}<\frac{\pi}{2}$, we have $\operatorname{Mi}\left(f, \Delta\left(\zeta, \varphi_{\varsigma}^{1}, \varphi_{\varsigma}^{2}\right)\right)<+\infty$, and if, further, there exists $a$ value $a \in \Omega$ and a set $N$ metrically dense on $\gamma$ such that $a \in C(f, \zeta, h(\zeta, \varphi))$ on at least one $h(\zeta, \varphi)$ at every $\zeta \in N$, then $f(z) \equiv a$.

Proof. According to Lemma, the set $M$ does not contain Plessner points of $f(z)$. It follows from Meier's theorem (see, for instance, [4], pp. 153-5) that $M$ must contain at least one Meier point of $f(z)$, so that the arc $r$ contains a subarc $\gamma_{0}$ almost all points of which are Fatou points of $f(z)$. Hence, the set $N$ contains a subset $N_{0}$, mes $N_{0}>0$, which consists of Fatou points of $f(z)$, and the corresponding angular limits of $f(z)$ are equal to $a$. Now theorem 1 follows from Lusin-Privalov's theorem.

As an immediate consequence of Plessner's theorem and of our lemma we obtain

Theorem 2. Let $f(z)$ be meromorphic in $D$. If at every point $\zeta$ of a set $M$ on $\Gamma$, mes $M>0$, the conditions (i), (ii) and (iii) of theorem 1 are valid and if, in addition, there exists a value $a \in \Omega$ such that $a \in C(f, \zeta, h(\zeta, \varphi))$ on at least one $h(\zeta, \varphi)$ at every $\zeta \in M$, then $f(z) \equiv a$.

4. In addition to theorems 1 and 2 we prove

Theorem 3. Let $\mu(r)$ be a positive, strictly increasing function on $0 \leq r<1$, $\lim _{r \rightarrow 1} \mu(r)=+\infty$. There exists a function $F(z)$ meromorphic in $D$ with the properties: (i) for almost all radii $h(\zeta, 0)$ we have $C(F, \zeta, h(\zeta, 0))=\{0\}$; (ii) every chord $h(\zeta, \varphi)$ contains a sequence of points $\left\{z_{n}\right\}, \lim _{n \rightarrow \infty} z_{n}=\zeta$, such that $\lim _{n \rightarrow \infty} \sigma\left(z_{n}, z_{n+1}\right)=0$ and $C\left(F, \zeta,\left\{z_{n}\right\}\right)=\{0\}$; (iii) the inequality $\left(1-|z|^{2}\right) \cdot \rho(F(z))<\mu(|z|)$ holds at all $z \in D$ with $|z|>r_{0}, 0<r_{0}<1$.

Proof. We start with the infinite product

$$
g(z)=\prod_{j=1}^{\infty}\left[1-\left(\frac{z}{1-\frac{1}{n_{j}}}\right)^{n_{j}}\right],
$$


the boundary behaviour of which has been studied by Bagemihl, Erdös and Seidel [2].

For any sequence of natural numbers $\left\{n_{k}\right\}$ satisfying the condition

$$
\lim _{k \rightarrow \infty} \frac{n_{k}}{n_{k-1}}=\infty, n_{1}>1 \text {, }
$$

the product (2) converges absolutely and uniformly on every disk $|z| \leq \rho$, $0<\rho<1$, and represents, therefore, a holomorphic function in $D$. If every zero $Z_{j}$ of $g(z)$ on the circumference $|z|=1-\frac{1}{n_{j}}$ is surrounded by a circle $r_{j}$ of radius $\frac{1}{j^{2} n_{j}}$, there exists an $j_{0}>0$ such that the interiors $\Gamma_{j}$ of $\gamma_{j}$ do not have common points for all $j \geq j_{0}$. So that the part of $D$ remaining after removing of $\Gamma_{j}, j \geq j_{0}$, is a domain $\Delta$.

The following properties of $g(z)$ are proved in [2]: (i) for any sequence of $\left\{n_{k}\right\}$ satisfying (3), the modulus $|g(z)|$ tends to $\infty$, as $|z| \rightarrow 1$, uniformly in $\Delta([2]$, p. 137); (ii) $g(z)$ possesses the radial limits at almost all points $\zeta=e^{i \theta} \in \Gamma([2]$, p. 139); (iii) if

$$
g_{k}(z)=\prod_{j \neq k}\left[1-\left(\frac{z}{1-\frac{1}{n_{j}}}\right)^{n_{j}}\right],
$$

then $\lim _{k \rightarrow \infty}\left|g_{k}\left(z_{k}\right)\right|=\infty$, where $z_{k}$ are zeros of $g(z)$ on $|z|=1-\frac{1}{n_{k}}, k=1,2, \cdots$ ([2], p. 142); (iv) for any $\mu(r)$ satisfying the conditions of theorem 3 one can find a sequence of odd numbers $\left\{n_{k}\right\}$ with $n_{k} \geq k n_{k-1}, k=1,2, \cdots$, such that $M(r, g)=\max _{|z|=r}|g(z)|<\mu(r)$ for all $r>r_{0}, 0<r_{0}<1$, ([2], p. 141).

Consider the derivative

$$
g^{-}(z)=-\sum_{\nu=1}^{\infty} \frac{n_{\nu} z^{n_{\nu}-1}}{\left(1-\frac{1}{n_{\nu}}\right)^{n_{\nu}}} g_{\nu}(z)
$$

we have, by the property (iii), that

$$
\lim _{k \rightarrow \infty}\left(1-\left|z_{k}\right|^{2}\right) \rho\left(g\left(z_{k}\right)\right)=\infty .
$$

We want to show that for any $\mu(r)$ in the conditions of theorem 3 one can choose a sequence of odd numbers $n_{k}$ satisfying

$$
\left(\sum_{j=1}^{k-1} n_{j}\right)^{2}<n_{k} \quad, \quad n_{1}>1 \text {, }
$$

such that for the corresponding function $g(z)$ defined by (2) 


$$
\left(1-|z|^{2}\right) \rho(g(z))<\mu(|z|)
$$

holds for all $z \in D,|z|>r_{0}, 0<r_{0}<1$.

For this end, let $k$ be chosen in such a way that

$$
1-\frac{1}{n_{k}} \leq|z|<1-\frac{1}{n_{k+1}}
$$

It is obvious that

$$
\begin{aligned}
& \left(1-|z|^{2}\right) \rho(g(z)) \leq\left(1-|z|^{2}\right)\left|g^{\prime}(z)\right| \leq \\
\leq & \left(1-|z|^{2}\right) \sum_{\nu=1}^{\infty} \frac{n_{\nu}|z|^{n_{\nu}-1}}{\left(1-\frac{1}{n_{\nu}}\right)^{n_{\nu}}} \prod_{j=1}^{\infty}\left[1+\left(\frac{|z|}{1-\frac{1}{n_{j}}}\right)^{n_{j}}\right] \leq \\
\leq & 2(1-|z|) M(|z|, g) \sum_{\nu=1}^{\infty} \frac{n_{\nu}|z|^{n_{\nu}-1}}{\left(1-\frac{1}{n_{\nu}}\right)^{n_{\nu}}} .
\end{aligned}
$$

The inequality (7) will lead to (5) if we prove that for all $z \in D,|z|>r_{0}$, and any sequence $\left\{n_{k}\right\}$ satisfying (4) the estimate

$$
\psi(z) \equiv 2(1-|z|) \sum_{\nu=1}^{\infty} \frac{n_{\nu}|z|^{n_{\nu}-1}}{\left(1-\frac{1}{n_{\nu}}\right)^{n_{\nu}}}<C
$$

is valid with a universal constant $C<+\infty$.

Using (4) and (6) and the obvious inequalities $\left(1-\frac{1}{n_{\nu}}\right)^{-n_{\nu}} \leqq 4, n_{1}>1$, $\nu=1,2, \cdots ; \log x<-(1-x)$ for $0<x<1$; and $x e^{-x}<1$ for $x>0$, we have

$$
\begin{aligned}
& \psi(z)<8(1-|z|)\left(\sum_{\nu=1}^{k-1} n_{\nu}+n_{k}+c_{1} \sum_{\nu=k+1}^{\infty} n_{\nu}|z|^{n_{\nu}}\right)< \\
< & 16(1-|z|) n_{k}+8 c_{1}(1-|z|) \sum_{\nu=k+1}^{\infty} n_{\nu} e^{-n_{\nu}(1-|z|)}< \\
< & 16+8 c_{1}(1-|z|) n_{k+1} e^{-n_{k+1}(1-|z|)}+8 c_{1} \sum_{\nu=k+2}^{\infty} n_{\nu} e^{-n_{\nu}(1-|z|)}< \\
< & 16+8 c_{1}+8 c_{1} \sum_{\nu=k+2}^{\infty} n_{\nu} e^{-\left(n_{\nu} / n_{k+1}\right)}<c_{2}+8 c_{1} \sum_{\nu=k+2}^{\infty} n e^{-\sqrt{n_{\nu}}}<C,
\end{aligned}
$$

where $c_{1}=\frac{1}{1-\frac{1}{n_{1}}}<+\infty$ and $k$ is sufficiently large, that proves (8).

The disks $\Gamma_{j}$ in the definition of the domain $\Delta$ may be regarded as non-Euclidean disks of radii $0\left(1 / j^{2}\right)$ (cf., [7], p. 396). Thus, the parts of a 
chord $h(\zeta, \alpha)$ that lie in $\Delta$ have the non-Euclidean distances tending to zero as $z$ approaches the boundary along $h(\zeta, \alpha)$.

Taking into account this fact and also the relation $\rho(f(z))=\rho(1 / f(z))$ which is valid for a function $f(z)$ meromorphic in $D$, we obtain the conclusion of theorem 3 for the function $F(z)=1 / g(z)$.

\section{REFERENCES}

[ 1 ] F. Bagemihl. Chordal limits of holomorphic functions at Plessner points. J. Sci. Hiroshima Univ. Ser. A, Div. 1, 30, 2 (1966), 109-115.

[2] F. Bagemihl, P. Erdös, W. Seidel. Sur quelques propriétés frontières des fonctions holomorphes definies par certains produits dans le cercle-unité. Ann. Ecole Norm. Sup. (3) 70 (1953), 135-147.

[3] E.F. Collingwood. On the linear and angular cluster sets of functions meromorphic in the unit circle. Acta Math., 91, 3-4 (1954), 165-185.

[4] E.F. Collingwood, A.J. Lohwater. The theory of cluster sets. Cambridge, University Press, 1966.

[5] E.P. Dolzhenko. On boundary uniqueness theorems and the behaviour of analytic functions near the boundary. (In Russian) Dokl. Akad. Nauk. SSSR, 129 (1959), 23-26.

[6] V.I. Gavrilov. On the distribution of values of non-normal meromorphic functions in the unit disk. (In Russian) Mat. Sb. 109 (n.s. 67) (1965), pp. 408-427.

[ 7 ] V.I. Gavrilov. Meromorphic functions in the unit disk with the prescribed growth of the spherical derivative (In Russian) Mat. Sb. 113 (n.s. 71) (1966), p.p. 386-404.

[ 8 ] K. Noshiro. Cluster sets. Berlin, Springer-Verlag, 1960.

Moscow University, USSR

Indian Institute of Technology, Bombay 